\title{
OPEN Comparative genomics reveals evolutionary loss of epiplakin in cetaceans
}

\author{
Peter Fuchs $^{1 凶}$, Corinne Drexler ${ }^{1}$, Sonia Ratajczyk ${ }^{1}$ \& Leopold Eckhart ${ }^{2 \bowtie}$
}

The adaptation of vertebrates to different environments was associated with changes in the molecular composition and regulation of epithelia. Whales and dolphins, together forming the clade cetaceans, have lost multiple epithelial keratins during or after their evolutionary transition from life on land to life in water. It is unknown whether the changes in keratins were accompanied by gain or loss of cytoskeletal adapter proteins of the plakin family. Here we investigated whether plakin proteins are conserved in cetaceans and other vertebrates. Comparative analysis of genome sequences showed conservation of dystonin, microtubule actin crosslinking factor 1 (MACF1), plectin, desmoplakin, periplakin and envoplakin in cetaceans. By contrast, EPPK1 (epiplakin) was disrupted by inactivating mutations in all cetaceans investigated. Orthologs of EPPK1 are present in bony and cartilaginous fishes and tetrapods, indicating an evolutionary origin of EPPK1 in a common ancestor of jawed vertebrates (Gnathostomes). In many vertebrates, EPPK1 is flanked by an as-yet uncharacterized gene that encodes protein domains homologous to the carboxy-terminal segment of MACF1. We conclude that epiplakin, unlike other plakins, was lost in cetaceans.

Epithelia line the surface of the skin and cavities in internal organs. The epithelium of the skin, the epidermis, forms the interface between the organism and its environment ${ }^{1,2}$. Changes in epidermal structure and composition represent key steps in the evolution of vertebrates, including the emergence of an efficient protection against water loss in tetrapods and evolution of specialized skin appendages, such as scales, claws, feathers and hair $^{3-5}$. The evolution of different lifestyles and diets has also led to modifications of internal epithelia of the lung, stomach, intestine and other organs ${ }^{6,7}$.

One of the key features of epithelial evolution was the adaptation of the cytoskeleton. Microfilaments and microtubules have been conserved with little or no variation whereas intermediate filaments have evolved specific compositions in many epithelial cell types with dynamic responses to stress ${ }^{6,8,9}$. Keratins are the characteristic intermediate filament proteins of epithelia. More than 50 keratins exist in humans with different heterodimers of type I and type II keratins being expressed in the epidermis, hair, skin glands, and stratified and simple epithelia of internal organs ${ }^{6,8,9}$. The diverse clades of vertebrates differ with regard to their sets of keratins. Recently, cetaceans (whales and dolphins) were shown to have a strongly reduced number of keratins, reflecting adaptations of the skin and perhaps other organs during their evolutionary transition from terrestrial to fully aquatic life ${ }^{10}$. Importantly, the quantitatively predominant proteins of the suprabasal epidermis of terrestrial mammals, i.e. keratins $\mathrm{K} 1, \mathrm{~K} 2$ and $\mathrm{K} 10$, are absent in cetaceans because of inactivating mutations in their genes ${ }^{10}$.

The function of the epithelial cytoskeleton depends also on large cytoskeletal linker proteins of the plakin family, which connect microfilaments, microtubules and intermediate filaments and thereby provide stress resistance and mechanical resilience ${ }^{11,12}$. Seven plakin proteins, not considering isoforms produced by alternative splicing, have so far been described in mammals: dystonin (DST)/bullous pemphigoid antigen 1 (BPAG1), desmoplakin (DSP), envoplakin (EVPL), epiplakin 1 (EPPK1), microtubule actin cross-linking factor 1 (MACF1), periplakin $(\mathrm{PPL})$, and plectin (PLEC) ${ }^{11,13-15}$. Plakins consist of multiple domains, including a plakin domain, plakin repeat domains, a growth-arrest-specific protein 2 (GAS2) domain, a calponin homology $(\mathrm{CH})$ domain, EF hand, a coiled-coil rod and a spectrin repeat rod ${ }^{12,13,16-18}$. However, these domains are not conserved in all members of the protein family. The signifying plakin domain, consisting of a Src-homology-3 (SH3) domain flanked by spectrin repeats, is present in all plakin proteins with the exception of epiplakin ${ }^{19,20}$. The latter has a unique structure as it contains multiple plakin repeat domains, which mediate binding to intermediate filaments, but no other domains ${ }^{21-25}$. At the gene level, EPPK1 is also unique because the entire coding sequence is present in

${ }^{1}$ Max Perutz Labs, Department of Biochemistry and Cell Biology, University of Vienna, Vienna Biocenter Campus (VBC), Vienna, Austria. ${ }^{2}$ Skin Biology Laboratory, Department of Dermatology, Medical University of Vienna, Vienna, Austria. ${ }^{\bowtie}$ email: peter.fuchs@univie.ac.at; leopold.eckhart@meduniwien.ac.at 


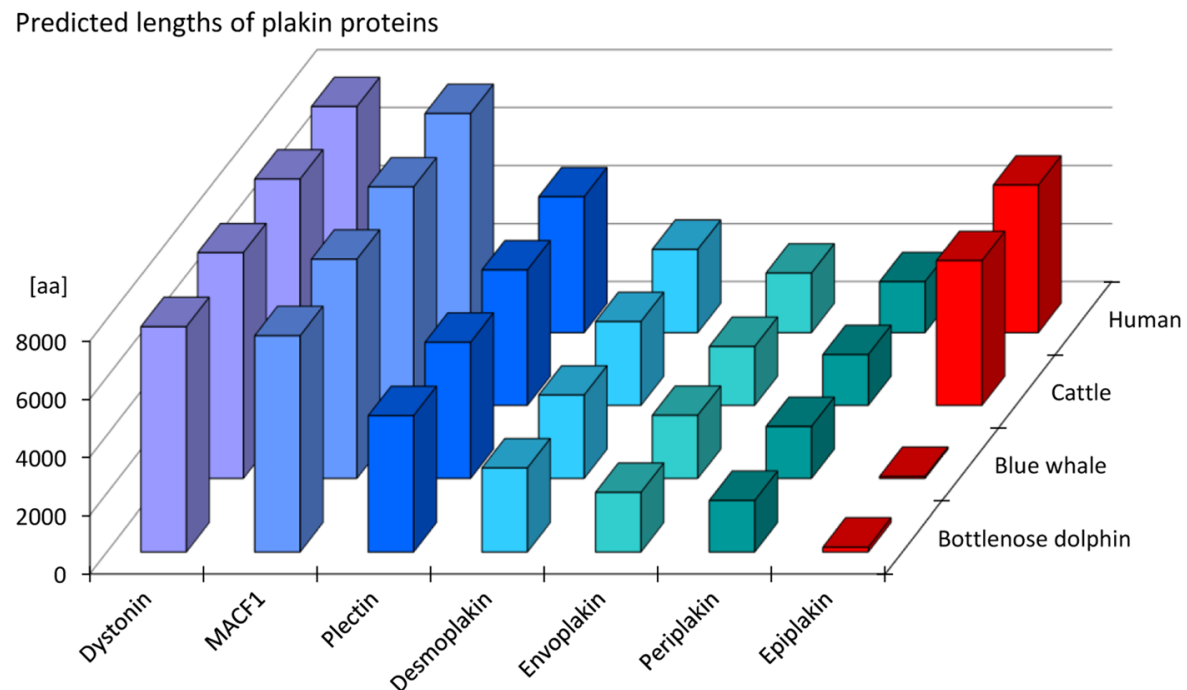

B Protein accession numbers (GenBank)

\begin{tabular}{|c|c|c|c|c|c|c|c|}
\hline Species & Dystonin & MACF1 & Plectin & Desmoplakin & Envoplakin & Periplakin & Epiplakin \\
\hline Human & NP_001361651 & NP_001380991 & NP_958782 & NP_004406 & NP_001307676 & NP_002696 & NP_112598 \\
\hline Cattle & XP_024839751 & XP_024842262 & XP_024857673 & NP_001179297 & NP_001192605 & XP_005224719 & XP_003586920 \\
\hline Blue whale & XP_036723642 & XP_036691322 & XP_036685833 & XP_036725975 & XP_036693372 & XP_036680364 & n.a. \\
\hline Bottlenose dolphin & XP_033720363 & XP_033693461 & XP_033699017 & XP_019800529 & XP_033702778 & XP_033696086 & n.a. \\
\hline
\end{tabular}

Figure 1. Size of plakin family proteins of land-dwelling mammals and cetaceans. (A) The predicted lengths of plakin family proteins are shown for human, cattle, blue whale and bottlenose dolphin. $a a$ amino acid residues. (B) GenBank accession numbers of the proteins that were analyzed in panel (A). For EPPK1 of the blue whale and the bottlenose dolphin, no prediction was available in GenBank. These proteins were predicted in this study by translating the region from the conserved start codon to the first in-frame stop codon, as shown in Supplementary Table S1. n.a. not applicable.

a single exon ${ }^{23,26}$ and consequently, alternative splicing that generates many isoforms of other members of this gene family, does not occur in EPPK1.

The aim of the present study was to determine whether evolutionary adaptions of the cytoskeleton in the epidermis and other epithelia were associated with changes in plakin genes. We show that epiplakin has undergone pseudogenization in cetaceans and, upon comparative genomics analysis of vertebrates, identify a previously uncharacterized plakin gene which is located besides EPPK1 in diverse species of fish and tetrapods and has been lost in a common ancestor of placental mammals.

\section{Results}

EPPK1 is inactivated by mutations in cetaceans. We investigated the published genome sequences of cetaceans for the presence of homologs of the seven human genes of the plakin family. Genes encoding MACF1, dystonin (DST)/BPAG1, plectin, desmoplakin, envoplakin and periplakin were conserved in the blue whale (Balaenoptera musculus), a representative of baleen whales (Mysticeti), and in the bottlenose dolphin, a representative of toothed whales (Odontoceti), whereas only unusually short coding sequences of epiplakin could be identified in these cetaceans (Fig. 1A,B; Supplementary Table S1). The lengths of the predicted plakin proteins of cetaceans were generally similar to those of their homologs in cattle and humans (Fig. 1A). By contrast, the lengths of the theoretical EPPK1 proteins, as determined by translation of the EPPK1 nucleotide sequence from the start codon until the first in-frame stop codon, were markedly smaller in the blue whale and bottlenose dolphin than in human and cattle (69 and 163 versus 5088 and 4997 amino acid residues, respectively) (Fig. 1A), indicating that epiplakin is not functional in cetaceans.

Alignment of nucleotide sequences revealed multiple frame-shift mutations in the $5^{\prime}$ region of the EPPK1 genes of the blue whale and dolphin (Fig. 2A), and many further frame-shifts and premature stop codons are present throughout the entire EPPK1 genes of these species. These mutations lead to truncated proteins that share only little sequence similarity with the amino-terminus of human epiplakin and contain only a small portion of the first plakin repeat domain (Fig. 2B). Similar truncations of the open reading frame of EPPK1 are present in 10 other species of cetaceans: white-sided dolphin (Lagenorhynchus obliquidens), orca (Orcinus orca), pilot whale (Globicephala melas), beluga whale (Delphinapterus leucas), narwhal (Monodon monoceros), baiji (Lipotes vexillifer), finless porpoise (Neophocaena asiaeorientalis), vaquita (Phocoena sinus), minke whale (Balaenoptera acutorostrata), and sperm whale (Physeter catodon) (Supplementary Fig. S1).

One single-nucleotide deletion (Fig. 2C, black asterisk) was present at the same position in both blue whale and dolphin, suggesting that this inactivating mutation has been inherited from a common ancestor. Comparison 
of nucleotide sequences flanking this site in other cetaceans showed the presence of the frame-shift relative to the human EPPK1 sequence in all cetaceans for which this segment of the gene could be identified in databases (Fig. 2C). As the mutation was determined by 11 independent genome sequencing projects, we can exclude that the sequence mismatch is caused by sequencing errors. In contrast to the conservation of the EPPK1 mutation in cetaceans, no frame-shift was detected at this site in human, cattle and the next land-dwelling or amphibious relative of cetaceans, the hippopotamus (Fig. 2C). Together, these data suggest that EPPK1 was inactivated in a common ancestor of cetaceans, that lived between 54 million years ago (estimated divergence of the lineages leading to cetaceans and hippopotamus ${ }^{27}$ ) and 33 million years ago (estimated divergence of the lineages leading to baleen whales and toothed whales ${ }^{27}$ ).

Comparative genome sequence analysis of mammals showed conservation of EPPK1 in all terrestrial species investigated. Analysis of the amino acid sequences encoded by EPPK1 genes revealed that the repeat structure is conserved through evolution (Supplementary Fig. S2).

In several species the coding sequence of EPPK1, which is between 6000 and 12,000 nucleotides long, contains one or few apparent mismatches with the inferred coding sequence (based on GenBank protein predictions). These sequence deviations may be caused by genuine mutations or by errors of sequencing or sequence assembly. For example, the protein prediction of cattle (Bos taurus) EPPK1 (accession number XP_003586920.3) is labelled as "low quality" in GenBank because of a single nucleotide mismatch between inferred coding sequence and the genome sequence. However, the apparent sequence deviation of EPPK1 in Bos taurus is found neither in the genome sequences of the closely related species, Bos indicus, nor in that of hybrid cattle (Bos indicus $\times$ Bos taurus) (Supplementary Table S2). The GenBank protein prediction of bovine EPPK1 is 4997 amino acid residues long, whereas translation of the sequence in the current genome sequence assembly would yield a protein of 2993 amino acid residues. EPPK1 of the hippopotamus could be identified only partially on a whole genome sequence contig (accession number PVJP02910133). According to the translation of this partial gene, the minimum length of the EPPK1 protein of the hippopotamus is 2290 amino acid residues (Supplementary Fig. S3). Our analysis suggests that EPPK1 genes encode long proteins with a characteristic plakin repeat domain-rich structure in mammals other than cetaceans. By contrast, the sequence between the EPPK1 start codon and the first in-frame stop codon does not encode a single plakin repeat domain in all cetaceans investigated (Fig. 2B and Supplementary Fig. S1), indicating a loss of function of epiplakin in this phylogenetic clade.

EPPK1 is conserved and flanked by a previously uncharacterized plakin gene in phylogenetically diverse Gnathostomes. Next we extended our comparative analysis of EPPK1 to non-mammalian species because EPPK1 homologs were recently reported also for the chicken ${ }^{28}$, Xenopus frogs and zebrafish ${ }^{29}$. We investigated the gene locus of EPPK1 in diverse vertebrates using published genome sequences available in GenBank. To this end we searched for EPPK1 and homologs of the genes that flank EPPK1 in the human genomes, i.e. PARP10 and PLEC, which are located on the $5^{\prime}$-side, and NRBP2 and PUF60, which are located on the $3^{\prime}$-side of EPPK1 (Fig. 3). All these genes are conserved in cattle and other placental mammals (Fig. 3). EPPK1 and neighboring genes were found in marsupials, monotremes, birds, reptiles and amphibians, suggesting that this gene arrangement and the presence of EPPK1 represents the ancestral condition of tetrapods. However, we identified an additional gene located between PLEC and EPPK1 in non-placental mammals and non-mammalian vertebrates (Fig. 3). This gene, tentatively named MACF1 carboxy-terminus-like (MACF1CTL), encodes a protein showing clear similarity to the carboxy-terminal segment of MACF1, including spectrin repeats, an EF hand and a GAS2 domain (Supplementary Fig. S4). Further genes are present between MACF1CTL and EPPK1 in the platypus (Fig. 3), possibly indicating a clade-specific chromosomal rearrangement in monotremes.

In zebrafish there are two PLEC orthologs, resulting from gene duplication in the course of a whole genome duplication during the evolution of teleost fishes ${ }^{30}$. EPPK1 is located besides one of the PLEC genes whereas a MACF1CTL gene is located besides the other PLEC homolog (Fig. 3), indicating that single copies of the latter genes have been conserved in zebrafish. Of note, the genes flanking PLEC and EPPK1 in tetrapods are located elsewhere in the zebrafish genome. In cartilaginous fishes (Chondrichthyes), which diverged around 470 million years ago from the lineage leading to bony fishes (Osteichthyes) including tetrapods ${ }^{27}$, we identified an EPPK1 gene in the genome of the thorny skate (Fig. 3). The gene is located close to PUF60, indicating partial conservation of the gene arrangement (synteny) relative to tetrapods. PLEC is located on another genome sequence scaffold, and a continuous sequence of the skate genome was not available at the time of our study. In another cartilaginous fish, the elephant shark, PLEC was flanked by MACF1CTL and by genes with similarities to plectin and epiplakin. EPPK1 homologs could be identified in the genomes of neither the sea lamprey, a representative of basal vertebrates, nor the sea squirt (Ciona intestinalis) a representative of basal chordates (Fig. 3). Together, these data suggest that EPPK1 is an evolutionarily ancient protein of Gnathostomes that has been conserved in diverse species but not in cetaceans (Fig. 4).

\section{Discussion}

The results of the present study suggest that EPPK1 originated more than 450 million years ago in a common ancestor of Gnathostomes and that it was inactivated by mutations in cetaceans. EPPK1 is expressed constitutively in the epidermis and in a stress-inducible manner in various epithelia of humans and the biomedical model species mouse, but its expression patterns and functions in other animals are not known. The high degree of conservation in many lineages of Gnathostomes suggests a critical function of EPPK1, whereas its loss in cetaceans indicates that its function is dispensable at least under some environmental and lifestyle-associated conditions. Indeed, EPPK1 knockout mice showed no obvious phenotype under standard laboratory housing conditions and only minor defects under stress conditions ${ }^{22,31-35}$, suggesting that EPPK1 is not essential for homeostatic life of mice and a possibly critical role of EPPK1 may be effective under as-yet unidentified circumstances. 
A

$\begin{array}{lllllllllllllllll}M & S & G & H & T & L & P & P & L & P & V & P & G\end{array}$

Human

Dolphin

Whale

Human

Dolphin

Whale

СTTTCTGTCCССTGCAGGACAGAGGTGGACCTCAGCAGGCTCTGCATAACCAGCCGTGTGTGATGAGTGGCCACACCTTGCCTCCTCTTCCCGTCCCAGG СTTTCCATCCCCACAGGGA------GCAGACGGTGGGCCCTGGCTGAGCAAGTTCTGTGCGATGAACAGCCACGTCT---CTCCTCTCAACCTCCTGGT СТСTCCGTCCCCACAGGGA------GCACA--GCGGTCCCCGGCTGAGCACGTTCTGCGCGATGAGCAGCCACGCCT---CTCCTCTCCACCTCCTGGT

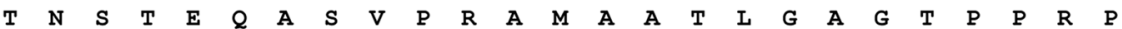
CACCAACAGCACAGAGCAGGCCAGTGTACCCAGAGCCATGGCAGCCACGCTGGGAGCCGGCACGCCCCCCAGGCCC

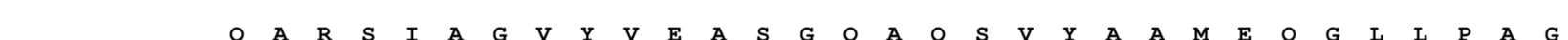

Human

Dolphin

CAGGCCAGGAGCATAGCTGGGGTTATGTGGAGGCCTCGGGCCAGGCCCAGAGTGTCTACGCCGCCATGGAGCAGGGCCTCCTGCCTGCTGGGC

-----CAGGCCAGGAGCCTCGCCGGCGTGTATGTGGAGGCCTCGGGCCAGGCCCAGCGTCTCTACGCTGTCATGAAGCAGGGTCTCCTGCCCTTTGGGC TCCACGCAGGCCAGGAGCATCGCGGG-GTGTACGTGGAGGCCTCGGGCCAGGCCCAGCGTCTCTGCGCTGTCGTGAGGCAGGGTCTCCTGCCCTTTGGGC

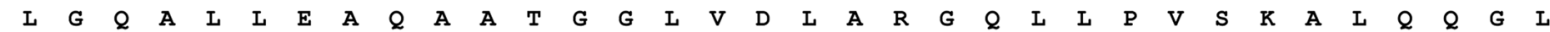
Human TCGGGCAGGCTCTGCTAGAGGCCCAGGCAGCCACTGGGGGCCTGGTGGACCTCGCCCGGGGCCAGCTGCTCCCTGTGTCCAAGGCCCTGCAGCAGGGTCT Dolphin TCGAGCTGGCTCTGCTGGAGGCCCAGGCGGCCCGGGC-------------AGGGCCAGCTGCTCCTCGTGTCTGAGGCCCTGCAGCAGGGCCT Whale TCGGGCTGGCTCTGCTGGAGGCCCAGGCGGCCACCGGGGGCCGCACGGACCCCGGGCAGGGCCAGCTGCTCCTCGTGTCTGAGGCCCTGGGGCAGG-CCT

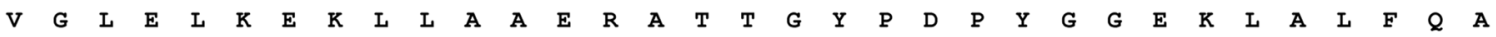

Human GGTGGGGCTGGAGCTGAAGGAGAAGCTGCTGGCCGCTGAGCGTGCCACTACGGGCTATCCTGACCCCTACGGCGGTGAGAAGCTGGCCCTCTTTCAGGCC Dolphin GGTGGGCCTGGAGCGGAAGGAGAAGCCGCTGGCCGCCGAGCACGCGGTCACTGGGTACCCTGA-CCCTCTGGGGGTGGGAAGCTGGCCCTCTTCCAGGCC Whale GGTGGGCCTGGAGCGGAAGAAGAAGC-GCTGGCCACTGAGCGCGCCGTCACCGGGTACCCTGA-CCCTCTGGGGGTGGGAAGCTGGCCCTCTTCCAGGCC

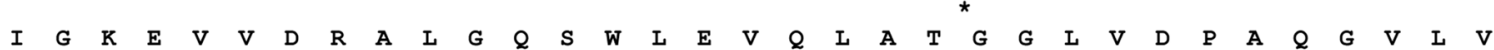

Human ATCGGGAAGGAGGTTGTGACAGGGCCTGGGGCAGAGCTGGCTGGAGGTCCAACTGGCCACTGGGGGCCTGGTGGACCCCGCCCAGGGAGTGCTCGTGG Dolphin ATAGGGAAGGAGGTTGTGGACAGGGCACTGGGGTGGAGCTGGCTGGAGGCCCAGGAGGCCACGGGGGGCCTGGTGGACCCCATCCAGGGCATGCGTGTGG Whale GTAGGGAAGGAGTTGTGGACAGGGCACTGGGCTGGAGCTGC--GGAGGCCCAGCTGGCCACGGGGGGCCTGGTGGACCCCATCCAGGGCGTCGCGTGG

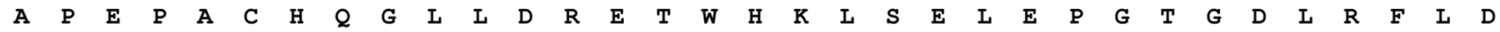
CCCCTGAGCCAGCCTGCCACCAGGGCCTCCTGGACCGGGAGACATGGCACAAGCTGTCAGAGCTTGAGCCTGGCACAGGTGACCTGCGCTTCCTCGACCC Dolphin CCCCTGAGCTGGCCTGCCAGCAAAGCCTCCTGGACCAGGAGACGTGGTGTGGGCTGTTGGAGCTTGGGCCCAGCTCAAGTGCCCCAGGCTTCCTGGACCC Whale CCCCTGAGCTGGCCTGCCAGCAAAGCCTCTTGGCCCAGGAGACATGGTGTGGGCTGTCGGAGCTTGGGCCCGGCTCAAGTGCCCCAGGCTTCCTGGACCC

B

Human epiplakin N-terminus Dolphin epiplakin Whale epiplakin

Human epiplakin N-terminus Dolphin epiplakin Whale epiplakin

Human epiplakin $\mathrm{N}$-terminus Dolphin epiplakin Whale epiplakin
Plakin repeat domain 1 -> VQLATGGLVDPAQGVLVAPEPACHQGLLDRETWHKLSELEPGTGDLRFLDPNTLERLTYHQLLERCVRAPGSGLALLPLKI GPGGHGGPGGPHPGHACGP*-0-0-0

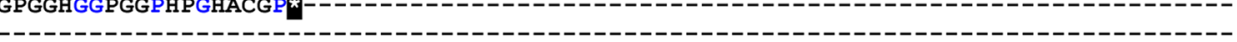
x
C

Human (Homo sapiens)

Cattle (Bos taurus)

Hippo (Hippopotamus amphibius)

Bottlenose dolphin (Tursiops truncatus)

White-sided dolphin (Lagenorhynchus obliquidens)

Orca (Orcinus orca)

Pilot whale (Globicephala melas)

Beluga whale (Delphinapterus leucas)

Narwhal (Monodon monoceros)

Baiji (Lipotes vexillifer)

Finless porpoise (Neophocaena asiaeorientalis)

Vaquita (Phocoena sinus)

Minke whale (Balaenoptera acutorostrata)

Blue whale (Balaenoptera musculus)

<- Plakin repeat domain 1 QARSIAGVYVEASGQAQSVYAAMEQGLLPAGLG MNSH-VSPLNLLVTNGTETAVVPETVKAVLGTGTPSGP---------QARS SAGVYVEASGQAQRLYAVMKQGLLPFGLE
MSSH-ASPLHLLVTSGTETAVVPETVTAVLGTGTPTPSGPQAEAAMSTQARSIAGCTWRPRARPSVSALS

Plakin repeat domain 1

OALLEAOAATGGLVDLARGQLLPVSKALQQGLVGLELKEKLLAAERATTGYPDPYGGEKLALFQAIGKEVVDRALGQSWLE LALLEAQAAPG-----RASCSSCLRPCSRAWWAWSGRRSRWPPSTRSLGTLTLWGWEAGPL-PGHREGGCGQGTGVELAG .

.

$\begin{array}{llllllllllllllllllllllllllll}A & T & T & G & Y & P & D & P & Y & G & G & E & K & L & A & L & F & Q & A\end{array}$

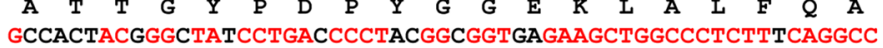
GCAGCCACTGGGTACCCTGATCCCTATGGGGGTCAGAAGCTGGCCCTCTTCCAGGCC GCCGTCACTGGGTACCCTGACCCСTATGGGGGTGGGAAGCTGGCCСTCTTCCAGGCC GCGGTCACTGGGTACCСTGA-СССTCTGGGGGTGGGAAGCTGGCССTCTTCCAGGCC GCGGTCACTGGGTACCCTGA-CCCTCTGGGGGTGGGAAGCTGGCCCTCTTCCAGGCC GCGGTCACTGGGTACCCTGA-СССTCTGGGGGTGGGAAGCTGGCССTCTTCCAGGCC GTGGTCACTGGGTACCCTGA-CCСTCTGGGGGTGGGAAGCTGGCCСTCTTCCAGGCC GTGGTCACTGGGTACCCTGA-СССTCTGGGGGTGGGAAGCTGGCССTCTTCCAGGCC GTGGTCACTGGGTACCCTGA-CССTCTGGGGGTGGGAAGCTGGCCСTCTTCCAGGCC GCAGTCACTGGGTACCCTGA-СCСTCTGGGGGTGGGAAGCTGGCССTCTTCCAGGCC GCGGTCACTGGGTACCCTGA-CССTCTGGGGGTGGGAAGCTGGCССTCTTCCAGGCC GCGGTCACTGGGTACCCTGA-CCCTCTGGGGGTGGGAAGCTGGCCCTCTTCCAGGCC GCCGTCACCGGGTACCCTGA-СССTCTGGGGGTGGGAAGCTGGCССTCTTCCAGGCC GCCGTCACCGGGTACCСTGA-СССTCTGGGGGTGGGAAGCTGGCССTCTTCCAGGCC * frameshift 
4Figure 2. Mutations disrupt the coding sequence of EPPK1 in cetaceans. (A) Alignment of partial nucleotide sequences of human, dolphin (Tursiops truncatus) and blue whale (Balaenoptera musculus) EPPK1. The translation of the coding sequence of human EPPK1 is shown above the nucleotide sequences. Red fonts indicate sequence identity in all 3 species. Dashes were added to optimize the alignment. Frameshift mutations are indicated by grey shading. A black asterisk indicates a frame-shift mutation that is conserved in dolphin and whale. (B) Alignment of amino acid sequences of the amino-terminal segment of human epiplakin (until the end of the first plakin repeat domain) and the translation products of dolphin (Tursiops truncatus) and blue whale (Balaenoptera musculus) EPPK1. The end of the sequence due to a stop codon is indicated by a white asterisk on black background. The plakin repeat domain 1 of human epiplakin is underlined. Red fonts indicate identity of residues in three species and blue fonts indicate conservation in two species. (C) Alignment of EPPK1 nucleotide sequences flanking a frame-shift mutation that is conserved in cetaceans [marked by the black asterisk in panel (A)]. Nucleotide sequences of homologous segments of EPPK1 from human, cattle, hippo and 11 species of cetaceans were aligned. Red fonts indicate sequence identity in all species. The translation of the human coding sequence is shown above the nucleotide sequences.

The water-to-land transition of cetaceans was associated with genetic adaptations that manifest in major anatomical and physiological changes. The epidermis of whales and dolphins is extremely thick and devoid of a granular layer. Many skin barrier-related genes have been lost ${ }^{28,36-38}$ and the number of keratin genes is greatly reduced in cetaceans ${ }^{10,39}$. In addition, genes related to kidney, heart, lung, eye, ear and nervous system development have been under positive selection in cetaceans $\mathrm{s}^{40-42}$, indicating adaptations of other organs. We hypothesize that the loss of EPPK1 in cetaceans was facilitated by the changes of the site of highest EPPK1 expression levels, which are the outer layers of the epidermis in other amniotes. However, EPPK1 must have important functions unrelated to the epidermal cornification because it originated and has been conserved in aquatic animals that lack a cornified epidermis (Fig. 3). In human liver, pancreas, colon and other organs, expression of EPPK1 is low under homeostasis ${ }^{23}$ but upregulated upon different types of stress ${ }^{22,35,43}$. The evolutionary history of EPPK1 suggests that its primordial function is related to stress responses. It remains to be investigated which epithelial stress response mechanisms depend on EPPK1 in most Gnathostomes but not in cetaceans. It will be interesting to find out whether the functions of EPPK1 are not required in cetaceans because the relevant stress to skin, liver, pancreas and colon is missing or if these types of stress are counteracted by mechanisms that substitute for EPPK1.

Epiplakin is unique among plakin family proteins because its structure is comprised of plakin repeat domains only. Our data demonstrate that the repeat organization of EPPK1 is evolutionarily conserved (Supplementary Fig. S2), confirming the results of a recent report on EPPK1 in a selection of model species ${ }^{29}$. The number of repeats differs among species and it is likely that even within species copy number variations exist, as exemplified by published data on human EPPK $1^{44}$ and mouse EPPK $1^{29}$. Experience with human and mouse EPPK1 gene has shown that the exact numbers of plakin repeat domains are difficult to determine by standard cloning and sequencing techniques and optimized long-range PCRs are required to ascertain the primary structure of EPPK1 in individuals of a broad range of species.

Previous data on the tandem arrangement of PLEC and EPPK1 genes in the human and mouse genomes and the observation that the repeat domains of EPPK1 show the highest sequence similarity to those of plectin ${ }^{17}$ have suggested that EPPK1 arose by partial tandem duplication of the PLEC gene. Our comparison of the PLEC and $E P P K 1$ gene loci across taxa of vertebrates reveals the existence of an as-yet uncharacterized gene, tentatively named MACF1CTL, located between PLEC and EPPK1 in fish, amphibians, reptiles, birds and basal mammals. These data suggest that the plakin repeat domain-coding part of $P L E C$, known from humans and mouse, was originally not a direct neighbor of EPPK1. Accordingly, MACF1CTL should be taken into account in hypotheses about the evolutionary origin of EPPK1.

$M A C F 1 C T L$ is the eighth member of the plakin family in mammals. Of note, a ninth plakin family member may be encoded by an as-yet uncharacterized envoplakin-like (EVPLL) in the human genome (GenBank gene ID: 645027). Due to gene loss, MACF1CTL is absent in placental mammals, including both cetaceans and humans. The protein domains of plectin are homologous to the amino-terminus of MACF1, whereas the protein domains of MACF1CTL are homologous to the carboxy-terminus of MACF1 (Supplementary Fig. S4). In some species, such as the zebra finch, the gene prediction algorithm used in GenBank has yielded a single gene (GenBank gene ID: 100223531) that connects the exons of PLEC and MACF1CTL. Further investigations of actual gene expression products are necessary to determine the structure and functions of MACF1CTL. Given the homology relationships of the protein domains of MACF1, PLEC and MACF1CTL, we put forward the hypothesis that PLEC and MACF1CTL evolved by the mechanism of gene fission ${ }^{45}$ from a single ancestral gene that was a copy of MACF1. The evolutionary origin of EPPK1 may have coincided with this gene recombination event.

In conclusion, our comparative genomics data suggest that $E P P K 1$ is an evolutionarily ancient gene of jawed vertebrates, which has been lost during the water-to-land transition of cetaceans. As EPPK1 is expressed at particularly high levels in the epidermis of terrestrial mammals, the loss of function of epiplakin may contribute to unique features of the epidermal cytoskeleton in cetaceans.

\section{Methods}

Ethics statement. Genes were investigated exclusively using sequences available in public databases. This research did not include human or animal subjects.

Identification and comparative analysis of plakin family genes. Orthology of genes was determined using the criteria of gene locus synteny and best reciprocal Basic Local Alignment Search Tool (BLAST) 


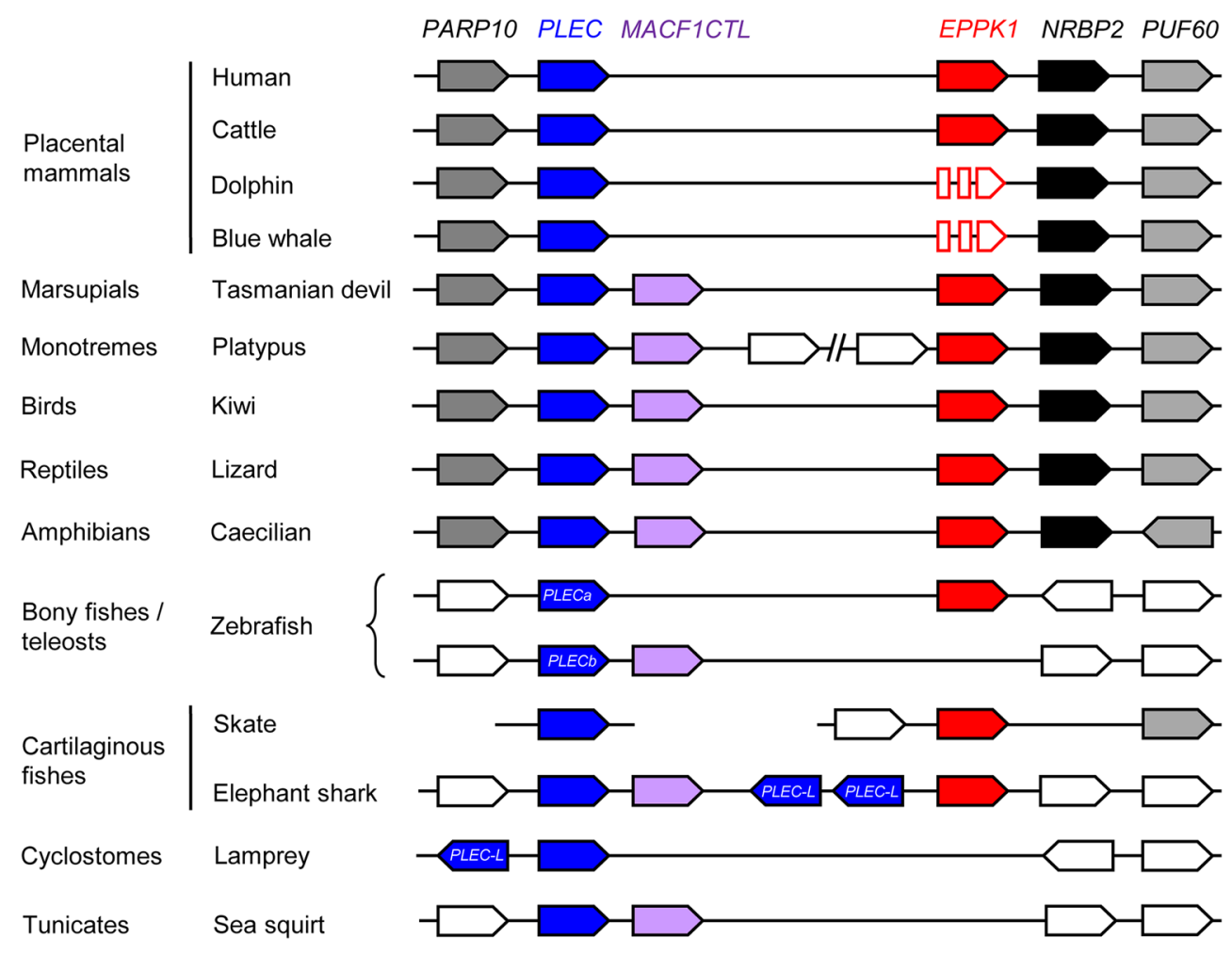

Figure 3. Comparison of PLEC and EPPK1 gene loci among chordates. Genes are schematically depicted by arrows pointing in the direction of transcription. Colors and grey shading mark orthologous genes of different species. White arrows represent genes that are not conserved across species. Disrupted arrows indicate the presence of inactivating mutations in the EPPK1 genes of cetaceans. The zebrafish has 2 PLEC genes (marked "a" and "b") due a teleost-specific whole-genome duplication. The identity of several genes in basal chordates is uncertain. Details about the genes are provided in Supplementary Table S1. A break in the horizontal line indicates that not all genes located between MACF1CTL and EPPK1 of the platypus are shown. The PLEC gene of the skate is located on a genomic sequence scaffold not yet placed into the current genome assembly. Note that EPPK1 is present in jawed vertebrates (Gnathostomata) but not in lamprey (Cyclostomata) and sea squirt (Urochordata); Species: Human (Homo sapiens), cattle (Bos taurus), blue whale (Balaenoptera musculus), bottlenose dolphin (Tursiops truncatus), tasmanian devil (Sarcophilus harrisii), platypus (Ornithorhynchus anatinus), kiwi (Apteryx rowi), caecilian (Rhinatrema bivittatum), zebrafish (Danio rerio), skate (Amblyraja radiata), lamprey (Petromyzon marinus), sea squirt (Ciona intestinalis). Genes: EPPK1, epiplakin 1; MACF1CTL, microtubule actin cross-linking factor 1 carboxy-terminus-like; PLEC, plectin; PLEC-L, PLEC-like.

hits of the encoded protein sequences. Nucleotide sequence were translated into amino acid sequences using the Translate tool at the Expasy website of the Swiss Institute of Bioinformatics (https://web.expasy.org/translate/). The domain structure of proteins was analyzed with the NCBI conserved domains tool (https://www.ncbi.nlm. nih.gov/Structure/cdd/wrpsb.cgi). Nucleotide and amino acid sequence were aligned with MUSCLE (https:// www.ebi.ac.uk/Tools/msa/muscle/). Amino acid sequence repeats in EPPK1 proteins were identified by dot plot analysis in which all parts of the protein were compared with all other parts of the same protein. Dot plots were generated by Dotmatcher (https://www.bioinformatics.nl/cgi-bin/emboss/dotmatcher) using default settings of "Window size over which to test threshold": 10 and "Threshold": 23, whereby input sequence 1 and input sequence 2 were identical. Origin and loss of genes during evolution were inferred from the pattern of presence or absence of orthologous genes in extant species according to the criterion of maximum parsimony. 


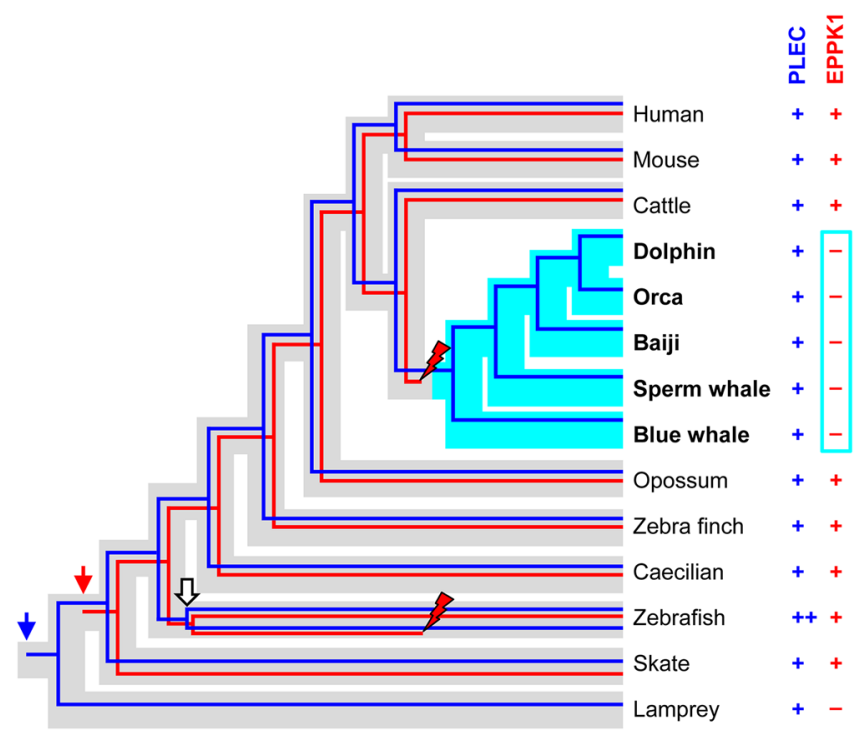

Figure 4. Schematic model of the origin and loss of EPPK1 during the evolution of vertebrates. The presence (+, 1 gene copy; ++, 2 gene copies) or absence (-) of intact PLEC and EPPK1 genes was used to infer the origin (vertical colored arrows) of PLEC (blue) and EPPK1 (red) genes and the loss of the capacity of EPPK1 to encode a functional plakin protein (red flash symbols) in cetaceans. The white arrow indicates a whole-genome duplication in teleosts. Phylogenetic trees of genes are shown within the species tree (grey for non-cetaceans, light blue for cetaceans).

\section{Data availability}

All data generated or analyzed during this study are included in this published article and its Supplementary Information files.

Received: 14 September 2021; Accepted: 6 January 2022

Published online: 21 January 2022

\section{References}

1. Proksch, E., Brandner, J. M. \& Jensen, J. M. The skin: An indispensable barrier. Exp. Dermatol. 17, 1063-1072. https://doi.org/10. $1111 / \mathrm{j} .1600-0625.2008 .00786 . x(2008)$.

2. Blanpain, C. \& Fuchs, E. Epidermal homeostasis: A balancing act of stem cells in the skin. Nat. Rev. Mol. Cell Biol. 10, $207-217$. https://doi.org/10.1038/nrm2636 (2009).

3. Alibardi, L. Adaptation to the land: The skin of reptiles in comparison to that of amphibians and endotherm amniotes. J. Exp. Zool. B. Mol. Dev. Evol. 298, 12-41. https://doi.org/10.1002/jez.b.24 (2003).

4. Schempp, C., Emde, M. \& Wolfle, U. Dermatology in the Darwin anniversary. Part 1: Evolution of the integument. J. Dtsch. Dermatol. Ges. 7, 750-757. https://doi.org/10.1111/j.1610-0387.2009.07193.x (2009).

5. Matsui, T. \& Amagai, M. Dissecting the formation, structure and barrier function of the stratum corneum. Int. Immunol. 27, 269-280. https://doi.org/10.1093/intimm/dxv013 (2015).

6. Bragulla, H. H. \& Homberger, D. G. Structure and functions of keratin proteins in simple, stratified, keratinized and cornified epithelia. J. Anat. 214, 516-559. https://doi.org/10.1111/j.1469-7580.2009.01066.x (2009).

7. Chen, L. et al. Large-scale ruminant genome sequencing provides insights into their evolution and distinct traits. Science 364, 1152. https://doi.org/10.1126/science.aav6202 (2019).

8. Eckhart, L., Ehrlich, F. \& Tschachler, E. A stress response program at the origin of evolutionary innovation in the skin. Evol. Bioinform. Online 15, 1-3. https://doi.org/10.1177/1176934319862246 (2019).

9. Jacob, J. T., Coulombe, P. A., Kwan, R. \& Omary, M. B. Types I and II keratin intermediate filaments. Cold Spring Harb. Perspect. Biol. 10, a018275. https://doi.org/10.1101/cshperspect.a018275 (2018).

10. Ehrlich, F. et al. Differential evolution of the epidermal keratin cytoskeleton in terrestrial and aquatic mammals. Mol. Biol. Evol. 36, 328-340. https://doi.org/10.1093/molbev/msy214 (2019).

11. Sonnenberg, A. \& Liem, R. K. Plakins in development and disease. Exp. Cell Res. 313, 2189-2203 (2007).

12. Hu, L., Huang, Z., Wu, Z., Ali, A. \& Qian, A. Mammalian plakins, giant cytolinkers: Versatile biological functions and roles in cancer. Int. J. Mol. Sci. 19, 974. https://doi.org/10.3390/ijms19040974 (2018).

13. Bouameur, J. E., Favre, B. \& Borradori, L. Plakins, a versatile family of cytolinkers: roles in skin integrity and in human diseases. J. Investig. Dermatol. 134, 885-894. https://doi.org/10.1038/jid.2013.498 (2014).

14. Jefferson, J. J., Leung, C. L. \& Liem, R. K. Plakins: Goliaths that link cell junctions and the cytoskeleton. Nat. Rev. Mol. Cell Biol. 5, 542-553 (2004).

15. Wiche, G. Role of plectin in cytoskeleton organization and dynamics. J. Cell Sci. 111, 2477-2486 (1998).

16. Wiche, G., Osmanagic-Myers, S. \& Castanon, M. J. Networking and anchoring through plectin: A key to IF functionality and mechanotransduction. Curr. Opin. Cell Biol. 32, 21-29. https://doi.org/10.1016/j.ceb.2014.10.002 (2015).

17. Fogl, C. et al. Mechanism of intermediate filament recognition by plakin repeat domains revealed by envoplakin targeting of vimentin. Nat. Commun. 7, 10827. https://doi.org/10.1038/ncomms10827 (2016).

18. Mohammed, F., Trieber, C., Overduin, M. \& Chidgey, M. Molecular mechanism of intermediate filament recognition by plakin proteins. Biochim. Biophys. Acta Mol. Cell Res. 1867, 118801. https://doi.org/10.1016/j.bbamcr.2020.118801 (2020). 
19. Suozzi, K. C., Wu, X. \& Fuchs, E. Spectraplakins: Master orchestrators of cytoskeletal dynamics. J. Cell Biol. 197, 465-475. https:// doi.org/10.1083/jcb.201112034 (2012).

20. Zhang, J., Yue, J. \& Wu, X. Spectraplakin family proteins-Cytoskeletal crosslinkers with versatile roles. J. Cell Sci. 130, $2447-2457$. https://doi.org/10.1242/jcs.196154 (2017).

21. Fujiwara, S. et al. Epiplakin, a novel member of the Plakin family originally identified as a 450-kDa human epidermal autoantigen. Structure and tissue localization. J. Biol. Chem. 276, 13340-13347 (2001).

22. Szabo, S. et al. Epiplakin attenuates experimental mouse liver injury by chaperoning keratin reorganization. J. Hepatol. 62, 13571366. https://doi.org/10.1016/j.jhep.2015.01.007 (2015).

23. Spazierer, D. et al. Epiplakin gene analysis in mouse reveals a single exon encoding a $725-\mathrm{kD}$ a protein with expression restricted to epithelial tissues. J. Biol. Chem. 278, 31657-31666. https://doi.org/10.1074/jbc.M303055200 (2003).

24. Spazierer, D., Raberger, J., Gross, K., Fuchs, P. \& Wiche, G. Stress-induced recruitment of epiplakin to keratin networks increases their resistance to hyperphosphorylation-induced disruption. J. Cell Sci. 121, 825-833 (2008).

25. Jang, S. I., Kalinin, A., Takahashi, K., Marekov, L. N. \& Steinert, P. M. Characterization of human epiplakin: RNAi-mediated epiplakin depletion leads to the disruption of keratin and vimentin IF networks. J. Cell Sci. 118, 781-793 (2005).

26. Takeo, N. et al. Structure and heterogeneity of the human gene for epiplakin (EPPK1). J. Investig. Dermatol. 121, 1224-1226 (2003).

27. Kumar, S., Stecher, G., Suleski, M. \& Hedges, S. B. TimeTree: A resource for timelines, timetrees, and divergence times. Mol. Biol. Evol. 34, 1812-1819. https://doi.org/10.1093/molbev/msx116 (2017).

28. Lachner, J. et al. An in vitro model of avian skin reveals evolutionarily conserved transcriptional regulation of epidermal barrier formation. J. Investig. Dermatol. 141, 2829-2837. https://doi.org/10.1016/j.jid.2021.04.029 (2021).

29. Ueo, D. et al. Intragenic copy number variation in mouse epiplakin 1 (Eppk1) and the conservation of the repeat structures in the lower vertebrates. J. Dermatol. Sci. 103, 186-189. https://doi.org/10.1016/j.jdermsci.2021.07.004 (2021).

30. Glasauer, S. M. \& Neuhauss, S. C. Whole-genome duplication in teleost fishes and its evolutionary consequences. Mol. Genet. Genom. 289, 1045-1060. https://doi.org/10.1007/s00438-014-0889-2 (2014).

31. Spazierer, D. et al. Epiplakin is dispensable for skin barrier function and for integrity of keratin network cytoarchitecture in simple and stratified epithelia. Mol. Cell. Biol. 26, 559-568 (2006).

32. Goto, M. et al. Elimination of epiplakin by gene targeting results in acceleration of keratinocyte migration in mice. Mol. Cell. Biol. 26, 548-558 (2006).

33. Kokado, M. et al. Increased fragility, impaired differentiation, and acceleration of migration of corneal epithelium of epiplakin-null mice. Investig. Ophthalmol. Vis. Sci. 54, 3780-3789. https://doi.org/10.1167/iovs.12-11077 (2013).

34. Shimada, H. et al. Epiplakin modifies the motility of the HeLa cells and accumulates at the outer surfaces of 3-D cell clusters. J. Dermatol. 40, 249-258. https://doi.org/10.1111/1346-8138.12076 (2013).

35. Wögenstein, K. L. et al. Epiplakin deficiency aggravates murine caerulein-induced acute pancreatitis and favors the formation of acinar keratin granules. PLoS One 9, e108323. https://doi.org/10.1371/journal.pone.0108323 (2014).

36. Holthaus, K. B., Lachner, J., Ebner, B., Tschachler, E. \& Eckhart, L. Gene duplications and gene loss in the epidermal differentiation complex during the evolutionary land-to-water transition of cetaceans. Sci. Rep. 11, 12334. https://doi.org/10.1038/s41598-02191863-3 (2021).

37. Strasser, B., Mlitz, V., Fischer, H., Tschachler, E. \& Eckhart, L. Comparative genomics reveals conservation of filaggrin and loss of caspase-14 in dolphins. Exp. Dermatol. 24, 365-369. https://doi.org/10.1111/exd.12681 (2015).

38. Abbas Zadeh, S. et al. Phylogenetic profiling and gene expression studies implicate a primary role of PSORS1C2 in terminal differentiation of keratinocytes. Exp. Dermatol. 26, 352-358. https://doi.org/10.1111/exd.13272 (2017).

39. Ehrlich, F. et al. Comparative genomics suggests loss of keratin K24 in three evolutionary lineages of mammals. Sci. Rep. 9, 10924. https://doi.org/10.1038/s41598-019-47422-y (2019)

40. McGowen, M. R., Gatesy, J. \& Wildman, D. E. Molecular evolution tracks macroevolutionary transitions in Cetacea. Trends Ecol. Evol. 29, 336-346. https://doi.org/10.1016/j.tree.2014.04.001 (2014).

41. Sharma, V. et al. A genomics approach reveals insights into the importance of gene losses for mammalian adaptations. Nat. Commun. 9, 1215. https://doi.org/10.1038/s41467-018-03667-1 (2018).

42. Huelsmann, M. et al. Genes lost during the transition from land to water in cetaceans highlight genomic changes associated with aquatic adaptations. Sci. Adv. 5, eaaw6671. https://doi.org/10.1126/sciadvaaw6671 (2019).

43. Yoshida, T. et al. Expression patterns of epiplakin1 in pancreas, pancreatic cancer and regenerating pancreas. Genes. Cells. 13, $667-678$ (2008).

44. Ishikawa, K. et al. Intragenic copy number variation within human epiplakin 1 (EPPK1) generates variation of molecular size of epiplakin. J. Dermatol. Sci. 91, 228-231. https://doi.org/10.1016/j.jdermsci.2018.05.008 (2018).

45. Kummerfeld, S. K. \& Teichmann, S. A. Relative rates of gene fusion and fission in multi-domain proteins. Trends Genet. 21, 25-30. https://doi.org/10.1016/j.tig.2004.11.007 (2005).

\section{Acknowledgements}

We thank Erwin Tschachler for helpful discussions. This work was supported by the Austrian Science Fund (FWF): P30310 (granted to P.F.) and P32777 (granted to L.E.).

\section{Author contributions}

P.F. and L.E. conceived the study, P.F., C.D. and L.E. performed bioinformatic analysis, P.F., C.D., S.R. and L.E. analyzed the results, P.F. and L.E. wrote the manuscript. All authors reviewed the manuscript.

\section{Competing interests}

The authors declare no competing interests.

\section{Additional information}

Supplementary Information The online version contains supplementary material available at https://doi.org/ 10.1038/s41598-022-05087-0.

Correspondence and requests for materials should be addressed to P.F. or L.E.

Reprints and permissions information is available at www.nature.com/reprints.

Publisher's note Springer Nature remains neutral with regard to jurisdictional claims in published maps and institutional affiliations. 
(c) (i) Open Access This article is licensed under a Creative Commons Attribution 4.0 International cc) License, which permits use, sharing, adaptation, distribution and reproduction in any medium or format, as long as you give appropriate credit to the original author(s) and the source, provide a link to the Creative Commons licence, and indicate if changes were made. The images or other third party material in this article are included in the article's Creative Commons licence, unless indicated otherwise in a credit line to the material. If material is not included in the article's Creative Commons licence and your intended use is not permitted by statutory regulation or exceeds the permitted use, you will need to obtain permission directly from the copyright holder. To view a copy of this licence, visit http://creativecommons.org/licenses/by/4.0/.

(C) The Author(s) 2022 